\title{
Incentive-based demand response in grid-connected microgrid using quasi-opposed grey wolf optimizer
}

\author{
Salil Madhav Dubey ${ }^{1}$, Hari Mohan Dubey ${ }^{1}$, Manjaree Pandit ${ }^{1}$ \\ ${ }^{1 *}$ Department of Electrical Engineering, Madhav Institute of Technology and Science, INDIA \\ "Corresponding Author: e-mail: salil.dubey3107@ gmail.com, Tel +91-8800610187 \\ ORCID iD: https://orcid.org/0000-0002-6193-294X (Salil Madhav Dubey), https://orcid.org/0000-0001-7237-4202 (Hari Mohan Dubey), \\ https://orcid.org/0000-0002-3984-5161 (Manjaree Pandit)
}

\begin{abstract}
The paradigm shifts in the electrical industry from demand-driven generation to supply-driven generation due to the incorporation of renewable generating sources is a growing research field. Implementing demand response in present-day distribution schemes is anattractive approach often adopted by microgrid (MiG) operator.This paper incorporates an incentivebased demand response (IBDR) method in a grid-connected microgrid (MiG) comprising of conventional generators (CGs), wind turbines (WTs), and solar PV units. The main aim is to collectively minimize the fossil fuel cost of CGs, lower the transaction cost of portable power from the grid, and maximize theMiG operator's profitafter implementing demand response. This multi-objective problem combining optimal economic load dispatch of MiG with an efficient demand-side response is solved using a proposed Quasi-opposed Grey Wolf Optimizer (QOGWO) algorithm. The effect of the proposed algorithm on demand-side management (DSM) is analyzed for two cases, (i) varying the value of power interruptibility (ii) varying the maximum limit of curtained power. Performance of QOGWO is compared with original GWO and a variant of GWO, Intelligent Grey Wolf Optimizer (IGWO). Results show the superior global search capability and complex constrained handling capability of QOGWO.
\end{abstract}

Keywords:Microgrid, Demand response, Grey wolf optimizer, Quasi opposition-based learning

DOI: http://dx.doi.org/10.4314/ijest.v13i2.1

Cite this article as:

Dubey S.M., Dubey H.M., Pandit M. 2021. Incentive-based demand response in grid-connected microgrid using quasi-opposed grey wolf optimizer. International Journal of Engineering, Science and Technology, Vol. 13, No. 2, pp. 1-14. doi: 10.4314/ijest.v13i2.1

Received: January 1, 2021; Accepted: February 2, 2021; Final acceptance in revised form: March 9, 2021

\section{Introduction}

The electrical power system is complicated due to various interacting factors that are either not adequately controlled or accurately predicted. Therefore, maintaining a reliable and secured operation in a power system is always a challenging task. To overcome these challenges, the concept of microgrid $(\mathrm{MiG})$ provides an alternative solution. MiG integrates microturbines, distributed energy resources (DERs), power storage system (PSS), and supply emergency power flexibly as it can work in either island or grid-connected mode.In islanded mode, MiG can be detached from the main grid in emergency but can still supply the power demand locally.In grid-connected mode, MiG can purchase electric power in case of a shortage or can sell excess energy to the grid leading to monetary gain.

Demand-Side Management (DSM) is a procedure to manage power mismatch between supply and demand by making changes in energy consumption patterns. It follows six major strategies: peak clipping, valley filling, strategic conservation, load building, 
Dubey et al./ International Journal of Engineering, Science and Technology, Vol. 13, No. 2, 2021, pp. 1-14

flexible load shape, and load shifting, to manage load efficiently over a scheduled period. These strategies can be implemented through demand response (DR). Here, customers are encouraged to reshape their energy consumption pattern by less energy utilization during peak hours or to move the time of energy utilization to off-peak duration. DR can be classified into two categories (i) Dispatchable or IBDR; wherecustomers are paid incentives for changing their energy consumption pattern and (ii) Non-dispatchable or price-based demand response (PBDR), where customers are charged with different tariff according to their electricity consumption time.

In recent years, researchers have shown more interest in MiG scheduling, including DR in it. A detailed review of various DR models for optimization related to energy management system isreported in various literature as presented in (Fallahi and Smith, 2017; Hussain and Gao, 2018; Jordehi, 2019; Khalili et al., 2019; Mizutani et al., 2018; Mohammed and Albadi, 2020; Robert et al., 2018; Sedighizadeh et al., 2018; Shariatzadeh et al., 2015). Researchers have proposed various energy management systems and models (EMS) to solve the generation scheduling problems either by using the traditional approach (Kiptoo et al., 2020; Mehdizadeh et al., 2018; Nwulu and Xia, 2017; SoltaniNejad Farsangi et al., 2018) or by using metaheuristic algorithms (Aghajani et al., 2015; da Silva et al., 2020; Esther et al., 2016; Jordehi, 2020; Kakran and Chanana, 2019; Niharika and Mukherjee, 2018; Nosratabadi and Hooshmand, 2020; Roy et al., 2019; Sarker et al.,2020; Shahryari et al., 2019) with the objective to either minimize operational cost or minimize both cost and emission.Grid-tied MiG with DR is seen as an excellent option by independent system operators (ISO) to achieve solutions to a large spectrum of modern-day electrical distribution problems.Futuristic MiG control systems are encouraged to include demand-side management (DSM) as an indispensable component (Imani et al., 2020;Ajoulabadi et al., 2020).

Various configurations of DR programs are investigated in (Imani et al., 2020), and a priority list of DRPs based on the weighting approach from microgrid operator (MO) considering MG's effects operation cost on the priority list is prepared.(Ajoulabadi et al., 2020)explores the benefit of PBDR programs in a MiG network and concludes that there is a significant improvement in the distribution system's power factor and the simultaneous reduction in network losses, operation cost, and peak demand.In (Kiptoo et al., 2020), mixed-integer linear programming (MILP) has been used for the techno-economic analysis of a stand-alone MiG. Here dynamic pricing approach as per the availability of renewable energy is utilized, and DSM has been investigated under different scenarios. In (Roy et al., 2019) a combination of ant lion optimization (ALO) and recurrent neural network (RNN) is proposed for EMS of grid-connected MiG.

Here DR has been carried out using recurrent neural network (RNN) for getting information of customer response, and ant lion optimization (ALO) is used for the energy scheduling. The optimum bidding price was calculated using information gap decision theory (IGDT), and price-based demand response was used to reduce the energy procurement in the competitive electricity market (Mehdizadeh et al., 2018). In (SoltaniNejad Farsangi et al., 2018), both price-based and incentive-based DR programs have been used and their impact on reducing operation cost of MiG in grid-connected and in island mode has been investigated using a twostage stochastic programming approach. Here it is observed that an incentive-based approach in grid-connected mode is found to be more effective in reducing operation cost. The IBDR has been utilized to minimize fossil fuel cost, cost associated due to transfer of power, and maximize the profit of MiG operators(Nwulu and Xia, 2017). Results show that incorporating DR in EMS is beneficial for the supply and the demand side of MiG from an economic point of view.

The multi-objective scheduling of MiG coupled with incentive-based DR has been investigated in (Aghajani et al., 2015; da Silva et al., 2020; Esther et al., 2016; Jordehi, 2020; Kakran and Chanana, 2019; Niharika and Mukherjee, 2018; Nosratabadi and Hooshmand, 2020;Olorunfemi and Nwulu, 2020; Roy et al., 2019; Shahryari et al., 2019)for minimization of operational cost and emissions released to the atmosphere. The augmented $\varepsilon$-constraint approach has been used to solve the multi-objective problem to minimize operation costs and emissions (Sedighizadeh et al., 2018). Here price-based DR is used to maintain system reliability during on-peak periods. In (Aghajani et al., 2015), multi-objective particle swarm optimization (MOPSO) has been utilized for minimizing operational cost and emission for MiG involving DR. Here, it is observed that the DR approach helps to mitigate power shortage caused due to the uncertainty of RES and is also useful in reducing operational cost and pollutant emission effectively.The benefit of using DR in grid-connected MiG after considering the uncertainties of demand, renewable power generation, and the market price is explored in (Jordehi, 2020). Here, particle swarm optimization (PSO) is used as the optimization algorithm.A multi-objective model is formulated in (Olorunfemi and Nwulu, 2020) to simultaneously minimize the annual cost of electricity production, minimize the carbon dioxide emission and maximize the customers' participation in the IBDRP to increase the customer benefit that comes with the program.An improved IBDR program for optimum multiobjective MiG scheduling is given in (Shahryari et al., 2019).

Improvisation in the DR program has been carried out by considering elasticity as a function of power consumption time, customer type, and peak load intensity. Here price-demand elasticity coefficients based on historical data and incentive payment model as a function of peak intensity and price-demand elasticity concept have been utilized for the intraday market. Finally, multi-objective group search optimization (MOGSO) is being used for minimizing both cost and emission in a competitive market. In (da Silva et al., 2020), non-dominated sorting genetic algorithm III (NSGA III) has been implemented for optimizing consumption cost of electricity, inconvenience cost of end consumers, and minimizing environmental pollution in a MiG, incorporating DR. Results show that DR helps to eliminate the overhead cost and reduce consumption cost of electricity. Bacterial foraging optimization algorithm (BFOA) (Esther et al., 2016), symbiotic organisms search (SOS) algorithm (Cheng and Prayogo, 2014)is used for DSM in a smart grid (Niharika and Mukherjee, 2018). Here energy management is carried out efficiently through 
load shifting approach. In (Nosratabadi and Hooshmand, 2020), day-ahead and intra-day generation planning is performed using the best DR programs selected based on the Normalized index of assessment strategy (NIAS) index in an industrial virtual power plant (VPP).

By the proposed methodology, DR programs benefit the industrial establishments by providing improved load shedding management and lower cost of operation. A home based EMS incorporate load shaping DR program for minimum electricity cost (Kakran and Chanana, 2019). Here, for the solution of scheduling problem, Particle Swarm Optimization (PSO) is implemented along with DR program for easy implementation.

In this paper, an incentive-based DR program is incorporated for the energy management problem operated under the gridconnected mode. As it is a highly constrained and complex optimization problem, a well-accepted population-based GWO with quasi opposition-based learning is proposed for solving it. The novelty of this paper can be summarized as follows:

- A new variant of GWO named QOGWO is proposed for the energy management problem of a microgrid

- Incorporating an incentive-based DR program in the system, its solution has been carried out using two variants of GWO to minimize total operating cost and to maximize the utility benefit.

- Effect of power interruptibility factor and daily curtailment limit are also analyzed from an economic point of view using the three algorithms for comparison.

As per the authors' knowledge, there is no work reported in the literature for the solution of DR program integrated with MiG using QOGWO. The developed model provides grid flexibility and simultaneously use DR to provide relief to the system. The rest of the paper is organized as follows: Section 2 presents the system's problem formulation. Section 3 explains the fundamental GWO and its variants that are used inthe simulation. Section 4 deals with the results obtained by simulation, and concluding remarks are presented in Section 5.

\section{Problem formulation}

\subsection{Grid-connected microgrid}

A trading scheme of power transfer is utilized here, in which electric power can either be purchased or sold to the grid to fulfill load demand over 24 hours. Here, the MiG considered for analysis combines three types of generating sources: Conventional generators (CGs), Wind turbines (WTs), and Solar PV units. Fuel cost associated with $\operatorname{CGs}\left(C_{i}\left(G_{i, t}^{C G}\right)\right)$ is given as (Nwulu and Xia, 2017):

$$
C_{i}\left(G_{i, t}^{C G}\right)=a_{i} G_{i, t}^{C G^{2}}+b_{i} G_{i . t}^{C G}
$$

The output power available from the WTs(Nwulu and Xia, 2017)is given as:

$$
\begin{aligned}
& G_{j, t}^{W}=0.5 \eta_{W} \rho C_{p} A v^{3} \\
& \text { where } \quad v=v_{\text {ref }}\left(\frac{h}{h_{\text {ref }}}\right)
\end{aligned}
$$

As the hourly solar power generation depends on solar radiation $\left(S_{t}\right)$ and area of $\mathrm{PV}$ array $\left(A_{P V}\right)$, it is given as:

$$
G_{k, t}^{S}=\eta_{S} A_{P V} S_{t}
$$

The cost associated with power exchange $C_{t r, t}\left(G_{t}^{t r}\right)$ between grid and MiGis represented as:

$$
C_{t r, t}\left(G_{t}^{t r}\right)=\left\{\begin{array}{cc}
\delta \cdot G_{t}^{t r} & G_{t}^{t r}>0 \\
0 & G_{t}^{t r}=0 \\
-\delta \cdot G_{t}^{t r} & G_{t}^{t r}<0
\end{array}\right.
$$

As the operating cost of RES is meager in comparison to the operating cost of CGs, hence it is not considered in the total operating cost calculation of microgrid (Nwulu and Xia, 2017). Therefore, the objective function that combines the fossil fuel cost of CGs and transactional cost of transferrable poweris given as:

$$
\min C=\sum_{t=1}^{24} \sum_{i=1}^{3} C_{i}\left(G_{i, t}^{C G}\right)+\sum_{t=1}^{24} C_{t r, t}\left(G_{i, t}^{t r}\right)
$$

\subsection{Demand response model}

This model assumes that customers are willing to alter the energy consumption pattern based on incentives paid for it by the utility. Accordingly, customers are classified based on willingness factor $\Psi \in(0,1) . \Psi=1$ signifies the most willing customer who wishes to change the energy consumption pattern (Nwulu and Xia, 2017). 
The cost incurred by a $l^{\text {th }}$ customer at time ' $t$ ', when he/she decreases his/her power consumption by time ' $g$ ' $\mathrm{kW}$ is given as:

$$
\mathbb{C}_{l, t}\left(\Psi_{l}, g_{l, t}\right)=k_{1, l} g_{l, t}{ }^{2}+k_{2, l} g_{l, t}\left(1-\Psi_{l}\right)
$$

Therefore, the customer benefit earned $\left(C B_{l, t}\right)$ by $l^{\text {th }}$ customer at time ' $t$ ' is represented as:

$$
C B_{l, t}=I_{l, t}-\mathbb{C}_{l, t}\left(\Psi_{l}, g_{l, t}\right)
$$

where ' $I_{l, t}$ ' is the incentive paid to $l^{\text {th }}$ customer by the utility for adjusting their energy consumption pattern at the time ' $t$ '.

Utility benefit $(U B)$ is the difference in cost associated with power curtailment to the utility and incentive paid to the customer. It is represented as:

$$
U B_{l, t}=\mu_{t} g_{l, t}-I_{l, t}
$$

The objective of $\mathrm{DR}$ in a MiG is to maximize the utility benefit $(U B)$ over 24 hours. It is represented as:

$$
\max U B=\sum_{t=1}^{24} \sum_{l=1}^{3} U B_{l, t}
$$

The grid-connected MiG with DR model has the objective to minimize the objective function $(F)$ which is given below (Nwulu and Xia, 2017):

$$
\min \mathrm{F}=\left[w\left\{\sum_{t=1}^{24} \sum_{i=1}^{3} C_{i}\left(G_{i, t}^{C G}\right)+\sum_{t=1}^{24} C_{t r, t}\left(G_{i, t}^{t r}\right)\right\}+(1-w)\left\{\sum_{t=1}^{24} \sum_{l=1}^{3} I_{l, t}-\mu_{t} g_{l, t}\right\}\right]
$$

subject to the following constraints:

$$
\begin{aligned}
& \sum_{i=1}^{3} G_{i, t}^{C G}+G_{i, t}^{t r}+G_{j, t}^{W}+G_{k, t}^{S}=P D_{t}-\sum_{l=1}^{3} g_{l, t} \\
& G_{i, \min }^{C G} \leq G_{i, t}^{C G} \leq G_{i, \max }^{C G} \\
& 0 \leq G_{j, t}^{W} \leq G_{j, \text { rated }}^{W} \\
& 0 \leq G_{k, t}^{S} \leq G_{k, \text { rated }}^{S} \\
& -G_{i, \max }^{t r} \leq G_{i, t}^{t r} \leq G_{i, \max }^{t r} \\
& -D R_{i} \leq G_{i, t+1}^{C G}-G_{i, t}^{C G} \leq U R_{i}
\end{aligned}
$$

$C B$ must be greater than zero to encourage customers for their active participation in demand response.

$$
\begin{aligned}
& \sum_{t=1}^{24} C B_{m} \geq 0 \text { for } m=1,2 \ldots . . l \\
& \sum_{t=1}^{24} C B_{m} \geq \sum_{t=1}^{24} C B_{m-1} \text { for } m=2 \ldots . l
\end{aligned}
$$

As DR is carried out daily, to make the model more practical from a market perspective, there is a fixed utility daily budget (UDB) for the incentive paid to customers. For utility benefit, the sum of incentive paid to customers for a day must be less than or equal to the allotted UDB. This constraint is represented as:

$$
\sum_{t=1}^{24} \sum_{l=1}^{3} I_{l, t} \leq U D B
$$

Similarly, it is assumed that there is a fixed power curtailment limit $(D L)$ for a day of each customer. The total power curtailed by a customer over 24 hours must be less than their specified $D L_{l}$ which is represented as:

$$
0 \leq \sum_{t=1}^{24} g_{l, t} \leq D L_{l}
$$

\section{Optimization Algorithms}

\subsection{Grey Wolf Optimizer}

Grey wolf optimization (GWO) comes under the umbrella of swarm intelligence-based algorithm proposed by Mirjalili et al. in 2014 (Mirjalili et al., 2014). Its analytical model imitates the leadership hierarchy of grey wolves and their organizedbehavior for hunting prey. GWO has four types of members named alpha, beta, delta, and omega. In the optimization process, the grey wolf position represents different position variables, and the distance from the prey to the wolf helps determine the fitness of objective function. Iteratively grey wolf will alter its position to move closer to the best position.

The disciplined group behavior of GWO is modeled in three phases of hunting mechanism as entrapment of prey, hunting of prey, and attacking the preyto reach prey by the shortest route.

\section{Entrapment of prey}

Like other metaheuristics, the initial population is generated randomly within the upper limit $(U L)$ and lower limit $(L L)$. In the first phase, if $\mathcal{X}(t)$ represents the current position of a wolf, then it will update its position to encircle prey positioned at $\mathcal{X}_{P}(t)$ by adjusting $\mathcal{A}$ and $\mathcal{C}$. The random vectors $r_{1}$ and $r_{2}$ each $\in(0,1)$ allow wolfs to adjust values of $\mathcal{A}$ and $\mathcal{C}$, respectively. The updated position of the grey wolf is represented as $\mathcal{X}(t+1)$ and the first phase of GWO is governed by (22)-(25).

$$
\chi(t+1)=\chi_{P}(t)-\mathcal{A} \times \mathcal{D}
$$

where

$$
\mathcal{A}=2 a r_{1}-a
$$




$$
\begin{aligned}
& \mathcal{D}=\left|\mathcal{C} \times \mathcal{X}_{P}(t)-\mathcal{X}(t)\right| \\
& \text { and } \mathcal{C}=2 r_{2}
\end{aligned}
$$

\section{Hunting of Prey}

To simulate the leadership hierarchy of grey wolves, it is assumed that alpha, beta, and gamma wolfs have better knowledge about the possible prey location. The Alpha wolf is supposed to be the nearest one (best solution); after that, the beta wolf and gamma wolf. The position of omega wolf will vary according to the current best position during the optimization process. The final position of the grey wolf is defined with respect to the position of alpha, beta, and delta in search space and represented as:

$$
\begin{array}{ll} 
& x(t+1)=\frac{1}{3} \times\left(x_{1}+x_{2}+x_{3}\right) \\
\text { where, } & x_{1}=x_{\alpha}(t)-\mathcal{A}_{1} \times \mathcal{D}_{\alpha}, x_{2}=x_{\beta}(t)-\mathcal{A}_{2} \times \mathcal{D}_{\beta}, x_{3}=x_{\delta}(t)-\mathcal{A}_{3} \times \mathcal{D}_{\delta} \\
\text { and } & \mathcal{D}_{\alpha}=\left|\mathcal{C}_{1} \cdot x_{\alpha}-x\right|, \mathcal{D}_{\beta}=\left|\mathcal{C}_{2} \cdot x_{\beta}-x\right|, \mathcal{D}_{\delta}=\left|\mathcal{C}_{3} . x_{\delta}-x\right|
\end{array}
$$

\section{Attacking the Prey}

This is the last phase of GWO, which occurs after the prey location has been identified, and then grey wolfs approach the prey to attack it. This approach method is mathematically simulated by varying parameter ' $\boldsymbol{a}^{\prime}$, according to (29).' $\boldsymbol{a}^{\prime}$ is a main control parameter of GWO that controls the exploration and exploitation phase. It decreases linearly from 2 to 0 over the course of iterations, which mimics the grey wolf's approaching behavior from the far end to the prey. Variation in values of ' $\boldsymbol{a}$ ' is mainly responsible for the exploration and exploitation of solutions in the search space.

$$
a=2-t\left(\frac{2}{T}\right)
$$

The steps of GWO involved during the optimization are as below:

- The position vector of the search agent is initialized randomly within its lower and upper limits.

- The fitness value of each search agent is evaluated on the basis of which three wolves are identified among the population; they are categorized as alpha, beta, and delta. They modify their position to catch the prey by $\mathcal{D}_{\alpha}, \mathcal{D}_{\beta}$ and $\mathcal{D}_{\delta}$ as in (28).

- $\quad$ Search agents update their position by (27).

- The above two steps (fitness calculation \& update mechanism) are repeated until termination criteria as specified isreached.

\subsection{Intelligent Grey Wolf Optimizer}

Intelligent Grey Wolf optimizer (IGWO) is a variant of GWO proposed in (Saxena et al., 2018), where two new mathematical frameworks are employed. (i) An opposition-based learning (OBL) approach for the better exploration and exploitation and (ii) use of the sinusoidal truncated function for variable ' $a$ '.

OBL approach helps to improve the convergence of population-based algorithm (Shaw et al., 2012; Tizhoosh, 2005; Xu et al., 2014). The OBL utilizes the opposite number and opposite points. The opposite number is a mirror point of the solution in terms of extreme points; the lower limit (LL), the upper limit (UL), and the search space center. It is represented as:

$$
\chi^{o}=L L+U L-\chi
$$

For point $P\left(\chi_{1}, \chi_{2}, \ldots \chi_{i} \cdot \chi_{d}\right)$, its opposite point $O P\left(\chi_{1}^{o}, \chi_{2}^{o} \ldots \chi_{i}^{0} \ldots \chi_{d}^{o}\right)$ expressed as (Mandal and Roy, 2013):

$$
\chi_{i}^{o}=L L_{i}+U L_{i}-\chi_{i} \forall \chi_{i} \in[L L, U L] \quad, i=1,2, \ldots . d
$$

where $d$ is the dimension of search space.

In IGWO, positions are initialized by considering half of the population as random and remaining by opposite population. The movement of the wolf is governed by the definition of parameter ' $a$ '. Here truncated sinusoidal function to control position and direction control is used, which is represented as:

$$
\begin{aligned}
a & =2 \times\left(1-\sin ^{2} \frac{\emptyset}{2}\right) \\
\text { where } \quad \emptyset & =\pi \times \frac{\text { Current iteration }}{\text { Maxiteration }}
\end{aligned}
$$

\subsection{Quasi-opposed Grey Wolf Optimizer}

Here, two mathematical frameworks are knit together to enhance the searching capability of GWO. They are (i) quasioppositional based learning concept and (ii) a nonlinear decreasing function ' $a$ '.

(i)Quasi-oppositional based learning

Quasi opposition-based learning (QOBL) is a modified version of OBL, and it is found to be more effective than OBL (Mandal 
and Roy, 2013; Rao, 2018). The quasi-opposite number $\left(\chi^{q o}\right)$, which is a number between the center of the search space and the opposite number is represented as:

$$
\chi^{q o}=\operatorname{rand}\left(\frac{L L+U L}{2}, \chi^{o}\right)
$$

Similarly, quasi opposite point $\left(\chi_{i}^{q o}\right)$ in the $d$ dimensional search space is represented as:

$$
\chi_{i}^{q o}=\operatorname{rand}\left(\frac{L L_{i}+U L_{i}}{2}, \chi_{i}^{o}\right) \quad \text { for } i=1,2,3 \ldots \ldots \ldots \ldots d
$$

(ii) Non-linear decreasing function ' $a$ '

Instead of using linearly decreasing ' $a$ ' as given in (29), a nonlinear decreasing control strategy is adopted in this proposed algorithm. This newly updated function' $a^{\prime}$ is represented below:

$$
a=2 \times\left(1-\left(\frac{\text { Current iteration }}{\text { Max iteration }}\right)^{m}\right)^{n}
$$

For simulation purposes, $m=3.98$ and $n=3.9$ are considered here, as suggested in (Suid et al., 2018). Three different patterns of parameter ' $a$ ' for GWO and its variants IGWO and QOGWO over 2500 iterations are illustrated in Figure 1.

3.4 Steps to implement QOGWO for Solution of incentive-based DR in microgrid

Step 1: Specify the population size ( variables $(\operatorname{dim} 2)$, customer cost function coefficients $\left(k_{1}, k_{2}\right)$, customer type factor $\left(\Psi_{l}\right)$, generator fuel coefficients $\left(a_{i}, b_{i}\right)$ and maximum iteration (T) as stopping criteria.

Step 2: Initialize population matrixes $\left(M^{\text {random }}\right)$ and $\left(G^{\text {random }}\right)$ of size [pop X diml] and [pop X dim2] respectively using randomization. Here, each element of $M^{\text {random }}$ signify the load curtailed by individual customers $\left(g_{l, t}\right)$ at a particular time ' $t$ ' and

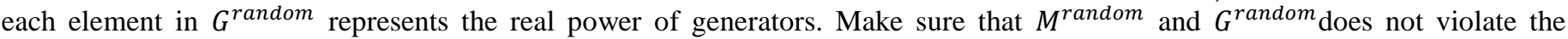
operational constraints given in (12)-(21).

$$
\begin{aligned}
& M^{\text {random }}=\left[\begin{array}{cccccc}
M 1^{1} & M 2^{1} & \cdots & \ldots & \cdots & \operatorname{Mdim} 1^{1} \\
M 1^{2} & M 2^{2} & \ldots & \ldots & \cdots & \operatorname{Mdim} 1^{2} \\
M 1^{3} & M 2^{3} & \cdots & \ldots & \ldots & \operatorname{Mdim} 1^{3} \\
\vdots & \vdots & \vdots & \vdots & \vdots & \vdots \\
\vdots & \vdots & \vdots & \vdots & \vdots & \vdots \\
M 1^{p o p} & M 2^{p o p} & \ldots & \ldots & \ldots & M \operatorname{dim} 1^{p o p}
\end{array}\right] \\
& G^{\text {random }}=\left[\begin{array}{cccccc}
G 1^{1} & G 2^{1} & \cdots & \ldots & \cdots & G \operatorname{dim} 2^{1} \\
G 1^{2} & G 2^{2} & \ldots & \ldots & \ldots & G \operatorname{dim} 2^{2} \\
G 1^{3} & G 2^{3} & \ldots & \ldots & \ldots & G \operatorname{dim} 2^{3} \\
\vdots & \vdots & \vdots & \vdots & \vdots & \vdots \\
\vdots & \vdots & \vdots & \vdots & \vdots & \vdots \\
G 1^{p o p} & G 2^{p o p} & \ldots & \cdots & \cdots & G \operatorname{dim} 2^{p o p}
\end{array}\right]
\end{aligned}
$$

Step3: Combine $M^{\text {random }}$ and $G^{\text {random }}$ to form a matrix $P^{\text {random }}$ of size $[p o p \mathrm{X}(\operatorname{dim} 1+\operatorname{dim} 2)]$ as given below

$$
P^{\text {random }}=\left[\begin{array}{ccccccc}
M 1^{1} & M 2^{1} & \ldots & M \operatorname{dim} 1^{1} & G 1^{1} & \ldots \ldots & G \operatorname{dim} 2^{1} \\
M 1^{2} & M 2^{2} & \ldots & M \operatorname{dim} 1^{2} & G 1^{2} & \ldots \ldots & G \operatorname{dim} 2^{2} \\
\vdots & \vdots & \vdots & \vdots & \vdots & \vdots & \vdots \\
\vdots & \vdots & \vdots & \vdots & \vdots & \vdots & \vdots \\
M 1^{p o p} & M 2^{p o p} & \ldots & M \operatorname{dim} 1^{p o p} & G 1^{p o p} & \ldots \ldots & G \operatorname{dim} 2^{p o p}
\end{array}\right]
$$

Step 4: Repeat step 2-3 to form population matrixes, $\left(M^{q o}\right)$ and $\left(G^{q o}\right)$ by using quasi-opposite learning as defined by (35) without violating (12-21). Matrix $P^{q o}$ thus, generated has size [pop X $\left.(\operatorname{dim} 1+\operatorname{dim} 2)\right]$.

$$
M^{q o}=\left[\begin{array}{cccccc}
M 1^{q o 1} & M 2^{q o 1} & \ldots & \ldots & \cdots & \operatorname{Mdim} 1^{q o 1} \\
M 1^{q o 2} & M 2^{q o 2} & \ldots & \ldots & \cdots & \operatorname{Mdim} 1^{q o 2} \\
M 1^{q o 3} & M 2^{q o 3} & \cdots & \cdots & \cdots & \operatorname{Mdim} 1^{q o 3} \\
\vdots & \vdots & \vdots & \vdots & \vdots & \vdots \\
\vdots & \vdots & \vdots & \vdots & \vdots & \vdots \\
M 1^{q o p o p} & M 2^{q o p o p} & \ldots & \cdots & \cdots & M \operatorname{dim} 1^{q o p o p}
\end{array}\right]
$$




$$
\begin{aligned}
& G^{q o}=\left[\begin{array}{cccccc}
G 1^{q 01} & G 2^{q o 1} & \cdots & \cdots & \cdots & \operatorname{Gdim} 2^{q o 1} \\
G 1^{q o 2} & G 2^{q o 2} & \cdots & \cdots & \cdots & \operatorname{Gdim} 2^{q o 2} \\
G 1^{q o 3} & G 2^{q o 3} & \cdots & \cdots & \cdots & \operatorname{Gdim} 2^{q o 3} \\
\vdots & \vdots & \vdots & \vdots & \vdots & \vdots \\
\vdots & \vdots & \vdots & \vdots & \vdots & \vdots \\
G 1^{q o p o p} & G 2^{q o p o p} & \cdots & \cdots & \cdots & G \operatorname{dim} 2^{q o p o p}
\end{array}\right] \\
& P^{q o}=\left[\begin{array}{ccccccc}
M 1^{q o 1} & M 2^{q o 1} & \ldots & M \operatorname{dim} 1^{q o 1} & G 1^{q o 1} & \ldots \ldots & G \operatorname{dim} 2^{q o 1} \\
M 1^{q o 2} & M 2^{q o 2} & \ldots & M \operatorname{Mim} 1^{q o} & G 1^{q o 2} & \ldots \ldots & G \operatorname{dim} 2^{q o 2} \\
\vdots & \vdots & \vdots & \vdots & \vdots & \vdots & \vdots \\
\vdots & \vdots & \vdots & \vdots & \vdots & \vdots & \vdots \\
M 1^{q o p o p} & M 2^{q o p o p} & \ldots & M \operatorname{dim} 1^{q o p o p} & G 1^{q o p o p} & \ldots \ldots & G \operatorname{dim} 2^{q o p o p}
\end{array}\right]
\end{aligned}
$$

Step 5: Calculate the fitness function value as mentioned in (11) for each vector in matrixes $P^{r a n d o m}$ and $P^{q o}$, formed in the above steps. Arrange all the vectors of $\left\{P^{\text {random }} \cup P^{q o}\right\}$ in ascending order of their fitness value and then select the vectors with best-fit values to form a new matrix $\left(P^{\text {initialize }}\right)$ of size $[p o p \mathrm{X}(\operatorname{dim} 1+\operatorname{dim} 2)]$.

Step 6: Find the first, second, and third best vectors of the matrix ( $P^{\text {initialize}}$ )which represent the positions of alpha, beta and gamma grey wolfs.

Step 7: The position of each grey wolf is modified according to the concept of entrapment, hunting, and attacking as discussed insection 3.1. Here each grey wolf position specifies a potential solution of problem.

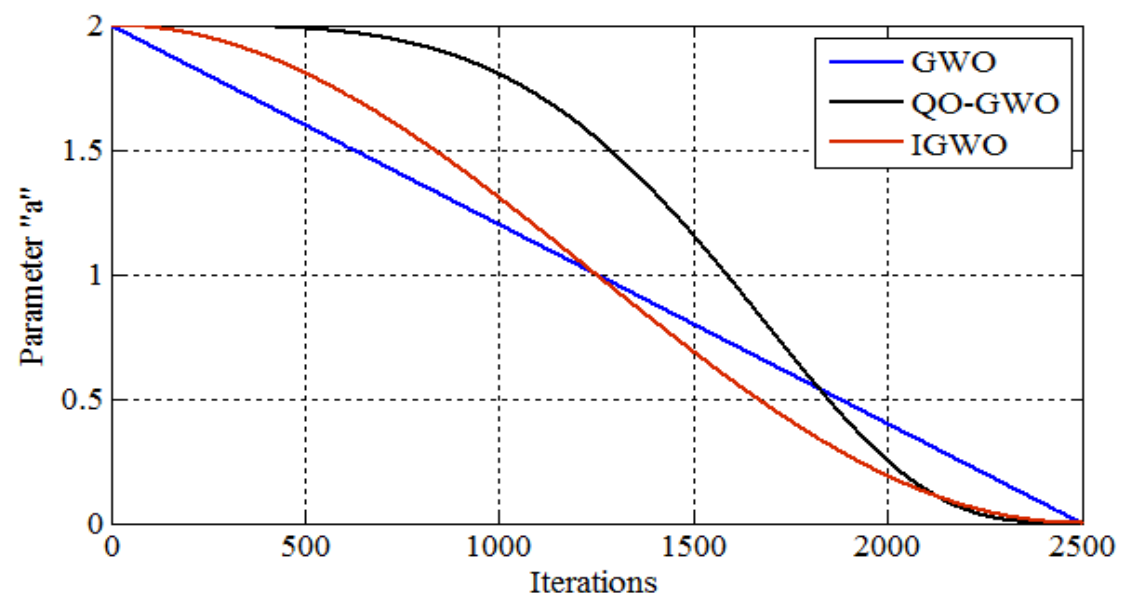

Figure 1 Variation in Parameter " $a$ " for different algorithms

Step 8: Check after each iteration if all the constraints mentioned in (12-21) are entirely fulfilled or not. If there is a violation of constraints, add a penalty to the fitness value to exclude the solution set's infeasible solution.

Step 9: Step 4 will be followed until the termination criteria are met. The QOGWO stops executing when termination criteria specified as maximum iteration $(T)$ is reached.

\section{Simulation results and Discussion}

\subsection{Explanation of Test case}

A grid-connected MiG system that combines three CGs, a solar PV unit, and a WT is considered here for optimum generation scheduling assuming three rural customers participating in DR. The details of cost coefficients of conventional (diesel) units $\left(\boldsymbol{a}_{\boldsymbol{i}}, \boldsymbol{b}_{\boldsymbol{i}}\right)$, minimum and maximum operational limits $\left(\boldsymbol{G}_{\boldsymbol{i}, \boldsymbol{m i n}}^{\boldsymbol{C G}}, \boldsymbol{G}_{\boldsymbol{i}, \boldsymbol{m a x}}^{\boldsymbol{C G}}\right)$ are listed in Table A1. Hourly load demand of the system $\left(\boldsymbol{P} \boldsymbol{D}_{\boldsymbol{t}}\right)$, values of power interruptibility factor $\left(\boldsymbol{\mu}_{\boldsymbol{t}}\right)$, and outputs of wind $\left(\boldsymbol{G}_{\boldsymbol{j}, \boldsymbol{t}}^{\boldsymbol{W}}\right)$ and solar units $\left(\boldsymbol{G}_{\boldsymbol{k}, \boldsymbol{t}}^{\boldsymbol{S}}\right)$ are presented in Table A2.The solar radiation and wind speed used in calculating the microgrid output are calculated from experimental data given in (Taxvinga et al., 2014) and the demand response model is incorporated in MATLAB, which gives simulated values.

The customer cost function coefficients $\left(k_{1, l}, k_{2, l}\right)$, customer type $\left(\Psi_{l}\right)$ and customer power limit on a daily basis $\left(D L_{l}\right)$ are listed in Table A3. For analysis purpose, it is assumed that (i) the maximum power limit that can be transferred between the main grid 
and $\mathrm{MiG}$ is assumed to be $12 \mathrm{KW}$, (ii) the microgrid operator knows $D L_{l}$ of each customer, which is then utilized to rank the customer to their willingness to curtail the electric power, (iii) The daily budget (UDB) of microgrid operator is taken as $\$ 500$. For simulation analysis purposes, $w=0.5$ is considered in (11), representing equal weightage to both the objectives. The above test case is analyzed for three cases, which are described in further subsections.

\subsection{Case 1: Analysis for a fixed value ofpower interruptibility factor $\left(\mu_{t}\right)$ and $D L_{l}$}

In this case, all three customers are assigned with equal values of power interruptibility factor $\left(\mu_{t}\right)$ and the fixed value of $\left(D L_{l}\right)$ as given in Table A2 \& A3, respectively.Table1 compiles the results obtained by three algorithms in terms of conventional power cost $(\$)$, transfer power cost $(\$)$, total operating cost $(\$)$, utility benefit $(\$)$, customer incentive $(\$)$, conventional power generated $(\mathrm{kWh})$, total power curtailed $(\mathrm{kWh})$ and transferred power $(\mathrm{kWh})$ for a particular day.

Here the total operating cost obtained by QOGWO is found to be about $143 \$(24 \%)$ lesser as compared to GWO and $44 \$(9 \%)$ lesser than IGWO. Therefore, it helps to maximize the profit of utility under IBDR. The utility benefit was increased by 114 (109 $\%$ ) compared to GWO and 23 \$ (12\%) compared to IGWO.

The QOGWO is found to be more efficient while managing the power economically. It takes less power from the grid to manage customer load demand, and the operational constraints are fully satisfied. The simulation outcome in terms of optimal generation schedule of CGs and hourly transferred power from the grid over 24 hours is presented in Figure 2. Also, the customers' curtained power and incentive on an hourly basis is illustrated in Figure 3.

\subsection{Case 2: Analysis for variation in $\mu_{t}$ and fixed value of $D L_{l}$}

In this case, impact analysis by the change in $\mu_{t}$ has been investigated. For analysis purpose, the interruptibility factor for three customers participating in DR is set at $\mu_{1 \mathrm{t}}=0.9 \mu_{\mathrm{t}}, \mu_{2 \mathrm{t}}=\mu_{\mathrm{t}}$ and $\mu_{3 \mathrm{t}}=1.1 \mu_{\mathrm{t}}$ respectively (Nwulu and Xia, 2017). The outcome of simulation results obtained by GWO and its variants are summarized in Table 2. After comparing the results with Case 1, it can be observed that there is a decrease in intensive for customer 1 , a minor change in incentive for customer 2 , and an increment in intensive for customer 3. The incentive compatibility constraint, as in (18) and (19),is satisfied. This case also similarly affects UB.

Table 1 Result after implementation of algorithms on the system

\begin{tabular}{|l|l|l|l|l|}
\hline S.No. & Parameters/Algorithms & GWO & IGWO & QOGWO \\
\hline $\mathbf{1}$ & Conventional power cost (\$) & 213.9999 & 229.65 & 225.18 \\
\hline $\mathbf{2}$ & Transfer power cost (\$) & 394.1765 & 280.05 & 240.03 \\
\hline $\mathbf{3}$ & OBJECTIVE 1 Total Operating Cost (\$) & $\mathbf{6 0 8 . 1 8}$ & $\mathbf{5 0 9 . 7 0}$ & $\mathbf{4 6 5 . 2 1}$ \\
\hline $\mathbf{4}$ & OBJECTIVE 2 Utility Benefit (\$) & $\mathbf{1 0 4 . 6 4}$ & $\mathbf{1 9 5 . 2 2}$ & $\mathbf{2 1 8 . 9 3}$ \\
\hline \multirow{4}{*}{} & Customer 1 & 32.37 & 59.99 & 67.32 \\
\cline { 2 - 5 } & Customer 2 & 34.22 & 62.19 & 71.67 \\
\cline { 2 - 5 } & Customer 3 & 38.05 & 73.05 & 79.93 \\
\hline \multirow{5}{*}{} & Customer Incentive (\$) & 337.66 & 253.86 & 228.51 \\
\hline \multirow{4}{*}{} & Customer 1 & 88.49 & 54.59 & 53.51 \\
\cline { 2 - 5 } & Customer 2 & 108.70 & 75.24 & 73.10 \\
\cline { 2 - 6 } & Customer 3 & 140.47 & 124.04 & 101.89 \\
\hline $\mathbf{6}$ & Conventional power (KWh) & 373.32 & 383.74 & 390.9464 \\
\hline $\mathbf{7}$ & Transferred power (KWh) & 66.8707 & 55.46 & 47.53 \\
\hline $\mathbf{8}$ & Total Power curtailed (KWh) & 78.71 & 79.70 & 80.42 \\
\hline
\end{tabular}



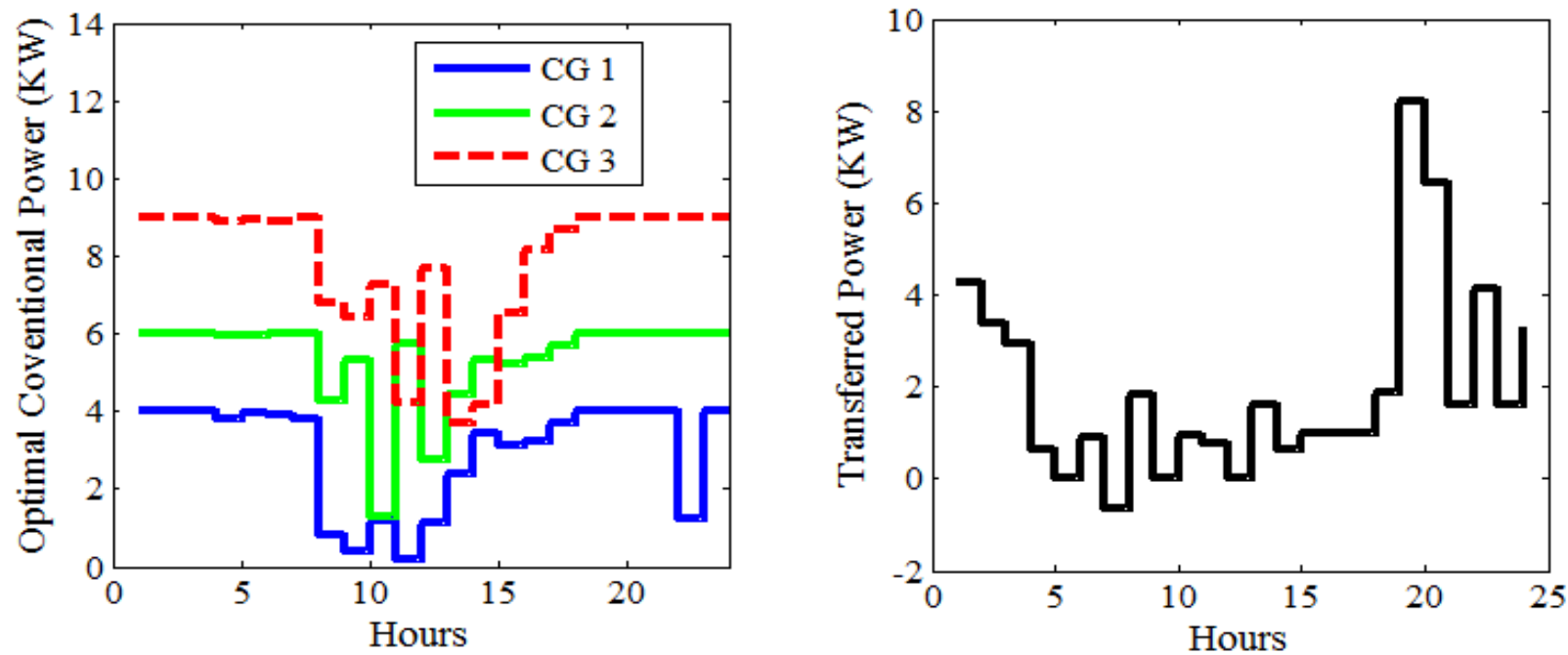

Figure 2 Optimum Generation of Conventional generators for QOGWO
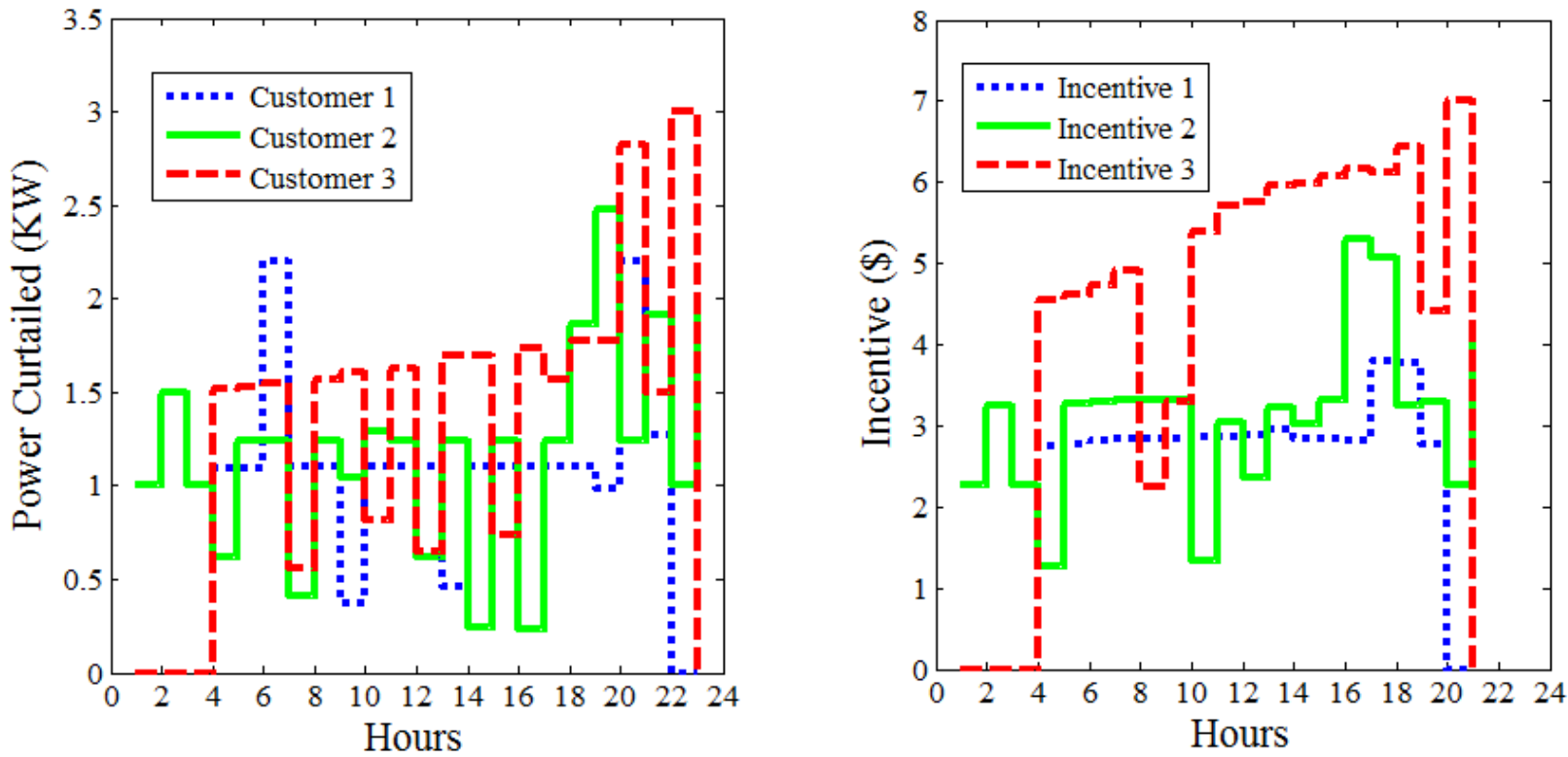

Figure 3 Power curtailed and Incentives for QOGWO

Table 2 Incentive to be paid and Company Profit after varying $\mu_{t}$

\begin{tabular}{|c|l|c|c|c|}
\hline S. No. & Parameters/Algorithms & GWO & IGWO & QOGWO \\
\hline $\mathbf{1}$ & Customer Incentive (\$) & 330.1044 & 254.0936 & 230.159 \\
\hline \multirow{3}{*}{} & Customer 1 & 77.4404 & 50.933 & 48.9532 \\
\cline { 2 - 5 } & Customer 2 & 107.4162 & 74.1346 & 72.3083 \\
\cline { 2 - 5 } & Customer 3 & 145.2478 & 129.026 & 108.8975 \\
\hline $\mathbf{2}$ & Utility Benefit (\$) & 106.277 & 203.898 & 240.626 \\
\hline \multirow{3}{*}{} & Customer 1 & 27.4749 & 53.4446 & 65.0352 \\
\cline { 2 - 5 } & Customer 2 & 34.4607 & 62.2216 & 72.4618 \\
\cline { 2 - 5 } & Customer 3 & 44.3414 & 88.2318 & 103.129 \\
\hline $\mathbf{3}$ & Total Power curtailed (KWh) & 78.37 & 79.07 & 80.12 \\
\hline
\end{tabular}


Table 3 Effect on the system after variation in $D L_{l}$

\begin{tabular}{|c|c|c|c|c|c|c|c|c|c|c|}
\hline \multirow{2}{*}{$\begin{array}{r}\text { S. } \\
\text { No. }\end{array}$} & \multirow{2}{*}{$\begin{array}{l}\text { Parameters/ } \\
\text { Algorithms }\end{array}$} & \multicolumn{3}{|c|}{$\underline{D L_{l}}(\mathrm{KW})$} & \multicolumn{3}{|c|}{$D L_{l}(\mathrm{KW})$} & \multicolumn{3}{|c|}{$\overline{D L_{l}}(\mathrm{KW})$} \\
\hline & & GWO & IGWO & QOGWO & GWO & IGWO & QOGWO & GWO & IGWO & QOGWO \\
\hline 1 & Conventional power cost $(\$)$ & 225.06 & 230.67 & 231.19 & 214.00 & 229.65 & 225.18 & 205.39 & 225.24 & 225.60 \\
\hline 2 & Transfer power cost $(\$)$ & 430.22 & 315.72 & 250.50 & 394.18 & 280.05 & 240.03 & 388.00 & 265.61 & 216.80 \\
\hline 3 & Total Operating Cost (\$) & 655.27 & 546.40 & 481.69 & 608.18 & 509.70 & 465.21 & 593.39 & 490.85 & 442.40 \\
\hline 4 & Total Power curtailed (KW) & 74.99 & 76.34 & 78.78 & 78.71 & 79.70 & 80.42 & 81.52 & 85.59 & 87.32 \\
\hline & Customer 1 & 19.43 & 20.27 & 22.27 & 20.87 & 19.65 & 20.86 & 19.83 & 20.08 & 21.09 \\
\hline & Customer 2 & 25.55 & 26.75 & 26.73 & 28.00 & 27.53 & 28.31 & 27.35 & 32.25 & 32.32 \\
\hline & Customer 3 & 30.01 & 29.32 & 29.78 & 30.83 & 33.53 & 31.25 & 34.34 & 33.26 & 33.90 \\
\hline 5 & Conventional power $(\mathrm{KW})$ & 376.10 & 377.47 & 390.52 & 373.32 & 383.74 & 390.95 & 371.40 & 384.67 & 388.65 \\
\hline 6 & Transferred power $(\mathrm{KW})$ & 67.81 & 65.09 & 49.60 & 66.87 & 55.46 & 47.53 & 65.99 & 48.64 & 42.93 \\
\hline
\end{tabular}

It is observed that the total power curtailed is found to be approximately the same as in case1, but the total utility benefit for all the three customers is found to be more. A similar trend is observed for all three algorithms considered under analysis. However, QOGWO is found to be most sensitive to change in $\mu_{t}$ with respect to the other two.

\subsection{Case 3: Analysis for a fixed value of power interruptibility factor $\left(\mu_{t}\right)$ and variation in $D L_{l}$}

In this case impact of variation in daily curtailment limit on power generation and its operational cost are investigated. For analysis purpose, the variation in power curtailment limit is considered to be more than $D L_{l}\left(\overline{D L_{l}}\right)$ and less than $D L_{l}\left(\underline{D L_{l}}\right)$ as compared to case 1. These limits are listed in Table A4. Change in $D L_{l}$ affects the energy generated by CGs. As the customers agree to curtail more load, the power generated by CGs decreases, thereby decreasing the operational cost.

Under this analysis scenario, the outcome of simulation by three algorithms is compiled and presented in Table 3. Comparing the two scenarios of power curtailed on demand-side, i.e., comparing $D L_{l}$ with $D L_{l}$. it can be observed that there is a decrement in the power curtailment of each customer. Curtailed load $\left(g_{l, t}\right)$ has decreased to $3.72 \mathrm{~kW}(5 \%), 3.37 \mathrm{~kW}(4 \%)$, and $1.65 \mathrm{~kW}(2 \%)$ for GWO, IGWO, and QOGWO algorithm, respectively, and the corresponding increase in the operating cost is about 47 \$ (8\%), 37 \$ $(7 \%), 16.5 \$(4 \%)$.

On the other hand, an increase in $D L_{l}$ to $\overline{D L_{l}}$, makes DR more productive by reducing the load demand for power generation resources. In this scenario, results show that there is an increase in curtailed power of $2.8 \$(4 \%), 5.9 \$(7 \%)$, and 7 \$ $(9 \%)$, and hence operational cost are reduced by 14.7 \$ (2\%), 18.8 \$ (4\%), and 22.8 \$ (5\%), as obtained for GWO, IGWO, and QOGWO respectively.

\section{Conclusion}

In this paper, a new variant ofGWO utilizes a quasi-opposition-based learning approach, and hence, named QOGWO, it has been proposed to manage a grid-connected MiG. For an incentive-based demand response model of MiG, optimal generation scheduling hasbeen carried out using QOGWO. To attract more customers to participate in demand response, a direct relationship between the power interruptibility factor and incentive given to each customer has also been incorporated in the model. The implication of power interruptibility factor and maximum daily curtailment limit on DR program are analyzed in the demand response model. For comparison and validation purposes, the above model is also implemented and simulated using classicalGWO and its recent variants called IGWO. Based on obtainedresults, the following conclusions are drawn:

- There is a direct relationship between the power interruptibility factor and the incentive given to each customer. Decrease in $\mu_{t}$, leads to a decrease in incentives paid to customers and vice-versa. Also, change in $\mu_{t}$ affects the utility benefit in a similar manner.

- The maximum power curtailment limit affects the total operating cost of the grid-connected MiG. More the power iscurtailed on the demand side, lesser will be the cost of operation and vice-versa.

- IBDR model is found to be effective in managing both the supply and the demand response for a MiG efficiently whensolved using metaheuristic techniques.

It can also be concluded that the prescribed demand response model can be solved using metaheuristic techniques like GWO, IGWO and QOGWO. Overall observation shows the dominance of QOGWO over IGWO as well as GWO in terms of solution quality. The simulated demand response model with the experimental data of microgridscan satisfy all constraints. The multiobjective demand response model is beneficial for all stakeholders. The distribution companies and customers are simultaneously benefited by incorporating the described IBDR model. 


\section{Acknowledgment}

The authors also sincerely acknowledge the financial support provided by AICTE-RPS project File No. 8228/RIFD/RPS/POLICY-1/2018-19 dated 20 March 2020.

\section{Nomenclature}

\begin{tabular}{|c|c|}
\hline$C_{i}\left(G_{i, t}^{C G}\right)$ & Cost of $i^{t h}$ conventional generator at time ' $t^{\prime}$ \\
\hline$a_{i}, b_{i}$ & Fuel coefficients of $i^{t h}$ conventional generator \\
\hline$G_{t}^{t r}$ & Transferred power from the grid at time ' $t$ ' \\
\hline$v$ & Velocity at any time \\
\hline$h$ & Height of wind turbine \\
\hline$G_{j, t}^{W}$ & Power output of $j^{t h}$ wind turbine at time ' $t^{\prime}$ \\
\hline$\rho$ & Density of air \\
\hline$A$ & Area swept by the wind turbine rotor \\
\hline$\eta_{S}$ & The efficiency of the solar PV generator/array \\
\hline$S_{t}$ & Solar irradiation incident on the solar PV array at time ' $t^{\prime}\left(\mathrm{kWh} / \mathrm{m}^{2}\right)$ \\
\hline$g_{l, t}$ & Load curtailed by $l^{\text {th }}$ customer at time ' $t^{\prime}$ \\
\hline$k_{1, l}, k_{2, l}$ & Cost function coefficients of $l^{\text {th }}$ customer \\
\hline$U B_{l, t}$ & Utility benefit function from $l^{\text {th }}$ customer at time ' $t$ ' \\
\hline$I_{l, t}$ & Incentive paid to $l^{\text {th }}$ customer at time ' $t^{\prime}$ \\
\hline$P D_{t}$ & Power demand at time ' $t$ ' \\
\hline$G_{j, \text { rated }}^{W}$ & Rated power output of $j^{\text {th }}$ wind turbine \\
\hline$G_{i, \max }^{t r}$ & Maximum transferrable power limit between grid and microgrid \\
\hline$U D B$ & Utility Daily Budget (500 \$) \\
\hline$a$ & Main control parameter of GWO.IGWO and QOGWO \\
\hline $\mathcal{A}, \mathcal{C}$ & Coefficient vectors \\
\hline$x(t)$ & Vector position of a grey wolf at $t$ time ' $t$ ' \\
\hline$x_{1}, x_{2}, x_{3}$ & Best position of alpha, beta and delta respectively. \\
\hline$t$ & Current iteration \\
\hline$G_{i, t}^{C G}$ & The power output of $i^{t h}$ conventional generator at time ' $t$ ' \\
\hline$C_{t r, t}\left(G_{t}^{t r}\right)$ & Cost of transferred power from the grid at time ' $t$ ' \\
\hline$\delta$ & Rate of transferred power from the grid $(5 \$ / \mathrm{kWh})$ \\
\hline$h_{\text {ref }}$ & Reference height of wind turbine \\
\hline$v_{\text {ref }}$ & Reference velocity at $h_{r e f}$ \\
\hline$\eta_{W}$ & The efficiency of the wind energy conversion system \\
\hline$C_{p}$ & Power coefficient of the wind turbine \\
\hline$G_{k, t}^{S}$ & The power output of $k^{t h}$ solar PV unit at time' $t^{\prime}$ \\
\hline$A_{P V}$ & Area of the PV array \\
\hline$\varepsilon_{l}$ & Customer type factor of $l^{\text {th }}$ customer normalized in $[0,1]$ \\
\hline $\mathbb{C}_{l, t}$ & Cost incurred after load curtailed by $l^{t h}$ customer at time' $t^{\prime}$ \\
\hline$C B_{l, t}$ & Customer benefit function of $l^{\text {th }}$ customer at time ' $t$ ' \\
\hline$w$ & Weight factor \\
\hline$\mu_{t}$ & Power interruptibility factor at time $t^{\prime}$ \\
\hline$i, \min , G_{i, \max }^{C G}$ & Minimum \& Maximum output limit of $i^{\text {th }}$ conventional generator \\
\hline$G_{k, \text { rated }}^{S}$ & Rated power output of $k^{t h}$ solar PV unit \\
\hline$U R_{i}, D R_{i}$ & Up ramp and Down ramp limit for $i^{t h}$ conventional generator \\
\hline$D L_{l}$ & Daily load curtailment limit for of $l^{\text {th }}$ customer \\
\hline LL, UL & Lower limit and Upper limit of a variable \\
\hline$\chi_{\mathrm{P}}(\mathrm{t})$ & Vector position of the prey at time ' $t$ ' \\
\hline$r_{1}, r_{2}$ & Random vectors $\epsilon[0,1]$ \\
\hline$x(t+1)$ & Updated position vector of a grey wolf at time ' $t+1^{\prime}$ \\
\hline$T$ & Maximumiteration \\
\hline
\end{tabular}




\section{Appendix}

Table A1: Conventional generator parameters

\begin{tabular}{|c|c|c|c|c|c|c|}
\hline CG No. & $\boldsymbol{a}_{\boldsymbol{i}}\left(\mathbf{\$} / \boldsymbol{k W h} \boldsymbol{h}^{\mathbf{2}}\right)$ & $\boldsymbol{b}_{\boldsymbol{i}}(\mathbf{\$} / \boldsymbol{k W h})$ & $\boldsymbol{G}_{\boldsymbol{i}, \boldsymbol{m i n}}^{\boldsymbol{C G}}(\mathbf{k W})$ & $\boldsymbol{G}_{\boldsymbol{i}, \boldsymbol{m a x}}^{\boldsymbol{C G}}(\mathbf{k W})$ & $\boldsymbol{U R}_{\boldsymbol{i}}$ & $\boldsymbol{D} \boldsymbol{R}_{\boldsymbol{i}}$ \\
\hline $\mathbf{1}$ & 0.06 & 0.5 & 0 & 4 & 3 & 3 \\
\hline $\mathbf{2}$ & 0.03 & 0.25 & 0 & 6 & 5 & 5 \\
\hline $\mathbf{3}$ & 0.04 & 0.3 & 0 & 9 & 8 & 8 \\
\hline
\end{tabular}

Table A2: Hourly load demand, power interruptibility factor, outputs of wind and solar units

\begin{tabular}{|c|c|c|c|c|}
\hline Hour & $\boldsymbol{G}_{\boldsymbol{j}, \boldsymbol{t}}^{\boldsymbol{W}}(\mathbf{k W})$ & $\boldsymbol{G}_{\boldsymbol{k}, \boldsymbol{t}}^{\boldsymbol{S}}(\mathbf{k W})$ & $\boldsymbol{P D}_{\boldsymbol{t}}(\mathbf{k W})$ & $\boldsymbol{\mu}_{\boldsymbol{t}} \mathbf{( \$ / k W h )}$ \\
\hline $\mathbf{1}$ & 7.56 & 0 & 31.83 & 1.57 \\
\hline $\mathbf{2}$ & 7.5 & 0 & 31.4 & 1.4 \\
\hline $\mathbf{3}$ & 8.25 & 0 & 31.17 & 2.2 \\
\hline $\mathbf{4}$ & 8.48 & 0 & 31 & 3.76 \\
\hline $\mathbf{5}$ & 8.48 & 0 & 31.17 & 4.5 \\
\hline $\mathbf{6}$ & 9.42 & 0 & 32.1 & 4.7 \\
\hline $\mathbf{7}$ & 9.82 & 0 & 32.97 & 5.04 \\
\hline $\mathbf{8}$ & 10.35 & 7.99 & 34.1 & 5.35 \\
\hline $\mathbf{9}$ & 10.88 & 10.56 & 37.53 & 6.7 \\
\hline $\mathbf{1 0}$ & 11.01 & 13.61 & 38.33 & 6.16 \\
\hline $\mathbf{1 1}$ & 10.94 & 14.97 & 40.03 & 6.38 \\
\hline $\mathbf{1 2}$ & 10.68 & 15 & 41.17 & 6.82 \\
\hline $\mathbf{1 3}$ & 10.42 & 14.78 & 39.67 & 7.3 \\
\hline $\mathbf{1 4}$ & 10.15 & 14.59 & 41.7 & 7.8 \\
\hline $\mathbf{1 5}$ & 9.67 & 13.56 & 42.1 & 8.5 \\
\hline $\mathbf{1 6}$ & 8.98 & 11.83 & 41.67 & 7.1 \\
\hline $\mathbf{1 7}$ & 8.37 & 10.17 & 40.7 & 6.8 \\
\hline $\mathbf{1 8}$ & 7.61 & 7.66 & 40.07 & 6.3 \\
\hline $\mathbf{1 9}$ & 6.7 & 0 & 38.63 & 5.8 \\
\hline $\mathbf{2 0}$ & 5.72 & 0 & 36.4 & 4.2 \\
\hline $\mathbf{2 1}$ & 7.21 & 0 & 34.1 & 3.8 \\
\hline $\mathbf{2 2}$ & 7.75 & 0 & 32.8 & 3.01 \\
\hline $\mathbf{2 3}$ & 7.88 & 0 & 32.5 & 2.53 \\
\hline $\mathbf{2 4}$ & 7.69 & 0 & 32 & 1.42 \\
\hline
\end{tabular}

Table A3: Rural Customer Details

\begin{tabular}{|c|c|c|c|c|}
\hline $\boldsymbol{l}$ & $\boldsymbol{k}_{\mathbf{1}, \boldsymbol{l}} \mathbf{( \$ \mathbf { k W } )}$ & $\boldsymbol{k}_{\mathbf{2}, \boldsymbol{l}} \mathbf{( \$ \mathbf { k W } )}$ & $\boldsymbol{\Psi}_{\boldsymbol{l}}$ & $\boldsymbol{D L}_{\boldsymbol{l}}(\mathbf{k W})$ \\
\hline $\mathbf{1}$ & 1.079 & 1.32 & 0 & 30 \\
\hline $\mathbf{2}$ & 1.378 & 1.62 & 0.45 & 35 \\
\hline $\mathbf{3}$ & 1.847 & 1.64 & 0.9 & 40 \\
\hline
\end{tabular}

Table A4 Variation in $D L_{l}$

\begin{tabular}{|c|c|c|c|}
\hline Customer No. & $\underline{\boldsymbol{D L}_{\boldsymbol{l}}}$ & $\boldsymbol{D L}_{\boldsymbol{l}}$ & $\overline{\boldsymbol{D L}_{\boldsymbol{l}}}$ \\
\hline Customer 1 & 27.5 & 30 & 32.5 \\
\hline Customer 2 & 32.5 & 35 & 37.5 \\
\hline Customer 3 & 35 & 40 & 45 \\
\hline Total & $\mathbf{9 5}$ & $\mathbf{1 0 5}$ & $\mathbf{1 1 5}$ \\
\hline
\end{tabular}




\section{References}

Aghajani, G.R., Shayanfar, H.A., Shayeghi, H., 2015. Presenting a multi-objective generation scheduling model for pricing demand response rate in micro-grid energy management. Energy Conversion and Management, Vol. 106, pp. 308-321. https://doi.org/10.1016/j.enconman.2015.08.059

Ajoulabadi, A., Ravadanegh, S.N., Mohammadi-Ivatloo, B., 2020. Flexible scheduling of reconfigurable microgrid-based distribution networks considering demand response program, Energy, Vol. 196, 117024.

https://doi.org/10.1016/j.energy.2020.117024

Cheng, M.Y., Prayogo, D., 2014. Symbiotic organisms search: A new metaheuristic optimization algorithm. Computers \& Structures, Vol. 139, pp. 98-112. https://doi.org/10.1016/j.compstruc.2014.03.007

da Silva, I.R.S., Rabêlo, R. de A.L., Rodrigues, J.J.P.C., Solic, P., Carvalho, A., 2020. A preference-based demand response mechanism for energy management in a microgrid. Journal of Cleaner Production, Vol. 255. https://doi.org/10.1016/j.jclepro.2020.120034

Esther, B.P., Krishna, K.S., Ravi, K., K, S.K., 2016. Demand side management using bacterial foraging optimization algorithm. Advances in Intelligent Systems and Computing, Vol. 433, pp. 21-29. https://doi.org/10.1007/978-81-322-2755-7

Fallahi, Z., Smith, A.D., 2017. Economic and emission-saving benefits of utilizing demand response and distributed renewables in microgrids. The Electricity Journal, Vol. 30, pp. 42-49. https://doi.org/10.1016/j.tej.2017.10.008

Hussain, M., Gao, Y., 2018. A review of demand response in an efficient smart grid environment. The Electricity Journal, Vol. 31, pp. 55-63. https://doi.org/10.1016/j.tej.2018.06.003

Imani, M.H., Ghadi, M.J., Ghavidel, S., Li, L., 2018. Demand response modeling in microgrid operation: a review and application for incentive-based and time-based programs, Renewable and Sustainable Energy Reviews, Vol. 94, pp. 486-499. https://doi.org/10.1016/j.rser.2018.06.017

Jordehi, A.R., 2019. Optimisation of demand response in electric power systems, a review. Renewable and Sustainable Energy Reviews, Vol. 103, pp. 308-319. https://doi.org/10.1016/j.rser.2018.12.054

Jordehi, A.R., 2020. Dynamic environmental $\square$ economic load dispatch in grid $\square$ connected microgrids with demand response programs considering the uncertainties of demand, renewable generation and market price. International Journal of Numerical Modelling: Electronic Networks, Devices and Fields, Vol. 34, e2798. https://doi.org/10.1002/jnm.2798

Kakran, S., Chanana, S., 2019. Optimal energy scheduling method under load shaping demand response program in a home energy management system. International Journal of Emerging Electric Power Systems, Vol. 20, pp. 1-11. https://doi.org/10.1515/ijeeps-2018-0147

Khalili, T., Nojavan, S., Zare, K., 2019. Optimal performance of microgrid in the presence of demand response exchange: A stochastic multi-objective model. Computers \& Electrical Engineering, Vol. 74, pp. 429-450.

https://doi.org/10.1016/j.compeleceng.2019.01.027

Kiptoo, M.K., Lotfy, M.E., Adewuyi, O.B., Conteh, A., Howlader, A.M., Senjyu, T., 2020. Integrated approach for optimal techno-economic planning for high renewable energy-based isolated microgrid considering cost of energy storage and demand response strategies. Energy Conversion and Management, Vol. 215. https://doi.org/10.1016/j.enconman.2020.112917

Mandal, B., Roy, P.K., 2013. Optimal reactive power dispatch using quasi-oppositional teaching learning based optimization. International Journal of Electrical Power \& Energy Systems, Vol. 53, pp. 123-134. https://doi.org/10.1016/j.ijepes.2013.04.011

Mehdizadeh, A., Taghizadegan, N., Salehi, J., 2018. Risk-based energy management of renewable-based microgrid using information gap decision theory in the presence of peak load management. Applied Energy, Vol. 211, pp. 617-630. https://doi.org/10.1016/j.apenergy.2017.11.084

Mirjalili, S., Mirjalili, S.M., Lewis, A., 2014. Grey wolf optimizer. Advances in Engineering Software, Vol. 69, pp. 46-61. https://doi.org/10.1016/j.advengsoft.2013.12.007

Mizutani, F., Tanaka, T., Nakamura, E., 2018. The effect of demand response on electricity consumption under the existence of the reference price effect: Evidence from a dynamic pricing experiment in Japan. The Electricity Journal, Vol. 31, pp. 16-22. https://doi.org/10.1016/j.tej.2018.01.004

Mohammed, N.A., Albadi, M.H., 2020. Demand response in electricity generation planning. The Electricity Journal, Vol. 33, 106799. https://doi.org/10.1016/j.tej.2020.106799

Niharika, Mukherjee, V., 2018. Day-ahead demand side management using symbiotic organisms search algorithm. IET Generation, Transmission \& Distribution, Vol. 12, pp. 3487-3494. https://doi.org/10.1049/iet-gtd.2018.0106

Nosratabadi, S.M., Hooshmand, R.A., 2020. Stochastic electrical energy management of industrial Virtual Power Plant considering time-based and incentive-based Demand Response programs option in contingency condition. International Journal of Emerging Electric Power Systems, Vol. 21, pp. 1-20. https://doi.org/10.1515/ijeeps-2019-0263

Nwulu, N.I., Xia, X., 2017. Optimal dispatch for a microgrid incorporating renewables and demand response. Renewable Energy, Vol. 101, pp. 16-28. https://doi.org/10.1016/j.renene.2016.08.026

Olorunfemi T.R., Nwulu N., 2020. Optimal Grid-Connected Microgrid Scheduling Incorporating an Incentive-Based Demand Response Program. In: Emamian S., Awang M., Yusof F. (eds) Advances in Manufacturing Engineering. Lecture Notes 
in Mechanical Engineering. Springer, Singapore. https://doi.org/10.1007/978-981-15-5753-8_56

Rao, R.V., 2018. Jaya: An Advanced Optimization Algorithm and its Engineering Applications, Springer, Singapore. https://doi.org/10.1007/978-3-319-78922-4

Robert, F.C., Sisodia, G.S., Gopalan, S., 2018. A critical review on the utilization of storage and demand response for the implementation of renewable energy microgrids. Sustainable Cities and Society, Vol. 40, pp. 735-745. https://doi.org/10.1016/j.scs.2018.04.008

Roy, K., Mandal, K.K., Mandal, A.C., 2019. Ant-lion optimizer algorithm and recurrent neural network for energy management of micro grid connected system. Energy, Vol. 167, pp. 402-416. https://doi.org/10.1016/j.energy.2018.10.153

Sarker, E., Seyedmahmoudian, M., Jamei, E., Horan, B., Stojcevski, A., 2020. Optimal management of home loads with renewable energy integration and demand response strategy, Energy. Vol. 210, 118602. https://doi.org/10.1016/j.energy.2020.118602.

Saxena, A., Soni, B.P., Kumar, R., Gupta, V., 2018. Intelligent Grey Wolf Optimizer - Development and application for strategic bidding in uniform price spot energy market. Applied Soft Computing, Vol. 69, pp. 1-13. https://doi.org/10.1016/j.asoc.2018.04.018

Sedighizadeh, M., Esmaili, M., Mohammadkhani, N., 2018. Stochastic multi-objective energy management in residential microgrids with combined cooling, heating, and power units considering battery energy storage systems and plug-in hybrid electric vehicles. Journal of Cleaner Production, Vol. 195, pp. 301-317. https://doi.org/10.1016/j.jclepro.2018.05.103

Shahryari, E., Shayeghi, H., Mohammadi-ivatloo, B., Moradzadeh, M., 2019. A copula-based method to consider uncertainties for multi-objective energy management of microgrid in presence of demand response. Energy, Vol. 175, pp. 879-890. https://doi.org/10.1016/j.energy.2019.03.129

Shariatzadeh, F., Mandal, P., Srivastava, A.K., 2015. Demand response for sustainable energy systems: A review, application and implementation strategy. Renewable and Sustainable Energy Reviews, Vol. 45, pp. 343-350. https://doi.org/10.1016/j.rser.2015.01.062

Shaw, B., Mukherjee, V., Ghoshal, S.P., 2012. A novel opposition-based gravitational search algorithm for combined economic and emission dispatch problems of power systems. International Journal of Electrical Power \& Energy Systems, Vol. 35, pp. 21-33. https://doi.org/10.1016/j.ijepes.2011.08.012

SoltaniNejad Farsangi, A., Hadayeghparast, S., Mehdinejad, M., Shayanfar, H., 2018. A novel stochastic energy management of a microgrid with various types of distributed energy resources in presence of demand response programs. Energy, Vol. 160, pp. 257-274. https://doi.org/10.1016/j.energy.2018.06.136

Suid, M.H., Ahmad, M.A., Ismail, M.R.T.R., Ghazali, M.R., Irawan, A., Tumari, M.Z., 2018. An improved sine cosine algorithm for solving optimization problems. Proceedings - 2018 IEEE Conference on Systems, Process and Control (ICSPC2018), pp. 209-213. https://doi.org/10.1109/SPC.2018.8703982

Tazvinga, H., Zhu, B., Xia, X., 2014. Energy dispatch strategy for a photovoltaic-wind-diesel-battery hybrid power system, Solar Energy. Vol. 108, pp. 412-420. https://doi.org/10.1016/j.solener.2014.07.025

Tizhoosh, H.R., 2005. Opposition-based learning: A new scheme for machine intelligence. Proceedings - International Conference on Computational Intelligence for Modelling Control and Automation (CIMCA 2005), International Conference on Intelligent Agents, Web Technologies and Internet Commerce (IAWTIC 2005), 28-30 November 2005, Vienna, Austria, 1, pp. 695-701. https://doi.org/10.1109/cimca.2005.1631345

Xu, Q., Wang, L., Wang, N., Hei, X., Zhao, L., 2014. A review of opposition-based learning from 2005 to 2012. Engineering Applications of Artificial Intelligence, Vol. 29, pp. 1-12. https://doi.org/10.1016/j.engappai.2013.12.004

\section{Biographical notes}

Salil Madhav Dubeyobtained his M.E. degree in Electrical Engineering from Madhav Institute of Technology and Science, Gwalior in 2020. He is pursuing PhD from Department of Energy Science and Engineering, IIT Bombay. His areas of research include microgrid operation, study of NI algorithms and its applications in power system.

Hari Mohan Dubeyobtained his M.E. degree in Electrical Engineering from Madhav Institute of Technology and Science, Gwalior in 2002 and Ph.D. degree from Rajiv Gandhi Proudyogiki Vishwavidyalaya, Bhopal, India, in 2017. He is with the Department of Electrical Engineering, M.I.T.S., Gwalior, (India). His areas of research include study of NI algorithms and their applications to different power system problems.

Manjaree Panditreceived her M.Tech. degree in Electrical Engineering from Maulana Azad College of Technology, Bhopal, India, in 1989 and the Ph.D. degree from Jiwaji University, Gwalior, India, in 2001. She is with the Department of Electrical Engineering, M.I.T.S., Gwalior. Her areas of interest are power system operation with integration of renewable energy sources, Nature Inspired optimization algorithms. 